\title{
Adjuvant Trastuzumab: Do We Finally Know How Long is Not Too Long?
}

\author{
Venkatraman Radhakrishnan ${ }^{1}$ \\ ${ }^{1}$ Department of Medical Oncology, Cancer Institute (WIA), Adyar, \\ Chennai, Tamil Nadu, India
}

Ind J Med Paediatr Oncol 2021;42:293-296.

Anti-human epidermal growth factor receptor 2 (antiHER2)-targeted treatment is the standard of care for treating HER2-positive breast cancer in the adjuvant, neoadjuvant, and metastatic setting. Trastuzumab was the first anti-HER2 therapy introduced and is the most widely used anti-HER2 drug.

Adjuvant trastuzumab has shown improvement in disease-free survival (DFS) and overall survival (OS) in HER2-positive breast cancer in multiple trials. ${ }^{1-4}$ These trials had given adjuvant trastuzumab for 1 year, and therefore 1 year has been the standard duration of prescribing trastuzumab.

Trials have examined giving adjuvant trastuzumab over a shorter duration (6 months, 3 months, or 9 weeks) instead of the standard 1-year duration. ${ }^{5-10}$ Shorter duration trastuzumab is attractive because of the reduced duration of treatment, decreased cardiac toxicity, and lesser financial toxicity.

However, randomized trials examining shorter duration of trastuzumab have given contradictory results and therefore the jury is still open on the duration of adjuvant trastuzumab. Gulia et al have tried to address this ambiguity through their recently published meta-analysis. ${ }^{11}$ What is unique about their paper is that they were able to extract individual patient data (IPD) from the Kaplan-Meier curves for the various adjuvant trastuzumab trials, using specialized software called the WebPlotDigitizer. The WebPlotDigitizer is a validated and reliable software for data extraction. ${ }^{12}$ An IPD meta-analysis is more robust than a trial-level meta-analysis. The meta-analysis included only randomized controlled trials (RCTs) that compared the shorter duration of trastuzumab ( $<1$ year) with 1 year of trastuzumab.

All trials comparing the shorter duration of adjuvant trastuzumab with 1-year trastuzumab must be noninferior in nature as the regulatory approval trials compared 1-year adjuvant trastuzumab to placebo. It will be difficult to show

\begin{abstract}
Address for correspondence Venkatraman Radhakrishnan, MD, DM, Department of Medical and Pediatric Oncology, Cancer Institute (WIA), Adyar, Chennai 600020, Tamil Nadu, India (e-mail: venkymd@gmail.com).
\end{abstract}

a superiority of a shorter duration of adjuvant trastuzumab to 1 -year trastuzumab. ${ }^{1-3}$ We also need to show that a shorter duration of adjuvant trastuzumab is not only noninferior but associated with lesser toxicity, lower mortality, and better quality of life compared with 1-year trastuzumab. There is no clear consensus on fixing the acceptable margin for noninferiority in an RCT. ${ }^{13}$ The studies included in the meta-analysis by Gulia et al had a noninferiority margin for the hazard ratio (HR) ranging from 1.15 to $1.53 .{ }^{11}$ Gulia et al took the median noninferiority margin for all the trials included in their meta-analysis and this was an HR of $1.3 .{ }^{11}$ That is if the upper limit of the $95 \%$ confidence interval (CI) of the HR for DFS and OS for the shorter duration of trastuzumab was less than 1.3, then they could conclude that the shorter duration of trastuzumab was noninferior to 1 -year trastuzumab. This would mean that a $30 \%$ reduced DFS or OS with shorter trastuzumab was acceptable. That is, patients have a $30 \%$ increased probability of disease relapse or death if they received a shorter duration of adjuvant trastuzumab compared with the standard 1-year adjuvant trastuzumab. Many patients and clinicians might not be willing to accept this increased risk. I would opine that for curable cancer in the adjuvant setting the noninferiority margin should be kept as low as possible. A noninferiority margin of 1.15 to 1.2 would have been appropriate for this meta-analysis. If the authors had chosen this noninferiority margin, then their meta-analysis would not have concluded that a shorter duration of adjuvant trastuzumab is not inferior to 1-year adjuvant trastuzumab.

PHARE and PERSEPHONE were trials that evaluated 6 months versus 1 year of adjuvant trastuzumab. ${ }^{9}{ }^{910}$ These trials were similar in design and outcomes, except that PHARE had a noninferiority limit for HR for DFS of 1.15 and PERSEPHONE had a limit of 1.25 . The trials had similar HR and 95\% CI (PHARE, HR: 1.08, 95\% CI: 0.93-1.25; PERSEPHONE, HR: 1.07, 95\% CI: 0.93-1.24). However, PHARE was a negative trial and
DOI https://doi.org/ $10.1055 / \mathrm{s}-0041-1735666$ ISSN 0971-5851
(C) 2021. Indian Society of Medical and Paediatric Oncology. This is an open access article published by Thieme under the terms of the Creative Commons Attribution-NonDerivative-NonCommercial-License, permitting copying and reproduction so long as the original work is given appropriate credit. Contents may not be used for commercial purposes, or adapted, remixed, transformed or built upon. (https://creativecommons.org/licenses/by-nc-nd/4.0/). Thieme Medical and Scientific Publishers Private Ltd. A-12, Second Floor, Sector -2, NOIDA -201301, India 
PERSEPHONE was a positive trial just because of the noninferiority margin they chose. The other trial comparing 6-month versus 1-year trastuzumab was the HORG trial and this trial took a noninferiority margin of 1.57 for DFS and it failed to show noninferiority. ${ }^{8}$

The HR for DFS for the five trials included in the IPD meta-analysis by Gulia et al was 1.14 (95\% CI: 1.03-1.25) and this was like the upper limit of CI reported by PHARE and PERSEPHONE. ${ }^{\text {-11 }}$ The absolute difference for 5-year DFS between the shorter duration and 1-year trastuzumab was $1.7 \%$ (85.4 vs. $87.1 \%$ ) and for OS was $1.1 \%$ (92.3 vs. 94.4\%) and this was not statistically significant. ${ }^{11}$ This means approximately 58 patients would need to be treated with 1-year trastuzumab to prevent 1 relapse and 90 patients would be needed to be treated with 1-year trastuzumab to prevent one death. Assuming a price of Indian Rupees 20,000 for a 440-mg vial of generic trastuzumab and one vial is used every 3 weeks (including the loading dose), the approximate cost of preventing one relapse would be Rs. 19,720,000 and for preventing one death would Rs. 30,600,000 with 1-year adjuvant trastuzumab. The high cost required to see the minimal benefits of 1-year trastuzumab might act as a deterrent for its use in the public health system funded by the taxpayer's money. The same might also apply to most of the patients who pay out of pocket and cannot afford the cost of treatment.

World Health Organization (WHO) defines an intervention to be cost effective if it costs less than three times the average per capita income per disability-adjusted life year (DALY) or quality-adjusted life-year (QALY). ${ }^{14}$ Therefore a formal cost-effectiveness analysis is required to see if 1-year adjuvant trastuzumab meets the WHO threshold of cost effectiveness. A recent study from India compared the cost effectiveness of 1 year, 6 months, and 9 weeks of adjuvant trastuzumab in breast cancer. ${ }^{15}$ The authors calculated the incremental cost per QALY gained with the use of adjuvant trastuzumab. The study concluded that 1-year trastuzumab is not cost effective in India at the current price. The 6-month and 9-week schedules were cost-effective with the most benefit seen with the 9 weeks schedule. A price reduction of 15 to $35 \%$ increased the probability of 1 -year trastuzumab use being cost effective to $90 \%$.

Gulia et al made an interesting observation in their results. ${ }^{11}$ When analyzed using a superiority design, 1-year trastuzumab was associated with a significantly better DFS (HR: 0.87; 95\% CI: 0.80-0.96) and OS (HR: 0.86; 95\% CI: 0.750.97). Instead of 1 -year adjuvant trastuzumab had the initial regulatory trials compared 6 months adjuvant trastuzumab to placebo, there would have been a possibility that future trials comparing 1 year versus 6 months of trastuzumab could have shown superiority of 1-year trastuzumab. This assumption is based on the results of the meta-analysis conducted by Gulia et al. ${ }^{11}$ In such a situation a shorter duration of adjuvant trastuzumab ( $<1$ year) might not be considered an appropriate treatment. Two-year adjuvant trastuzumab was compared with 1 year, and this was not shown to be superior. ${ }^{16}$
When dissecting trials that used adjuvant trastuzumab for 6 months (three trials) versus those that used adjuvant trastuzumab for 9 to 12 weeks (three trials), it was observed that trials that used 9 to 12 weeks of trastuzumab failed to show noninferiority for DFS and OS compared with 1-year trastuzumab. The HRs for DFS and OS (9-12-week vs. 1-year trastuzumab) were 1.27 (95\% CI: $1.07-1.51$ ) and 1.25 (95\% CI: 0.96-1.63), respectively. The HRs for DFS and OS (6-month vs. 1-year trastuzumab) were 1.10 (95\% CI: 0.99-1.23) and 1.14 (95\% CI: 0.99-1.32), respectively. Therefore, noninferiority could only be demonstrated for DFS and not OS for 6-month versus 1-year adjuvant trastuzumab. These results suggest that 9 to 12 weeks of trastuzumab is inadequate and 6 months of adjuvant trastuzumab cannot replace 1-year trastuzumab if OS is our endpoint. I would opine that we should not lump all short-duration trastuzumab in one group, it might not be like comparing apples and oranges, but it is like comparing different breeds of apples. Overall, taken together all short-duration regimens ( 6 months, 12 weeks, and 9 weeks), results show noninferiority. However, when considered individually, only the 6-month regimen showed noninferiority and not the 12 weeks or 9 weeks regimens.

The meta-analysis showed a $3 \%$ reduction in symptomatic congestive heart failure with a shorter duration of trastuzumab versus 1-year trastuzumab (3.9 vs. 6.9\%, $p<$ 0.001). ${ }^{11}$ Asymptomatic left ventricular ejection fraction decline was lower in the shorter trastuzumab group compared with the 1-year trastuzumab (5 vs. 7\%, $p=0.049$ ). These findings indicate that a shorter duration of trastuzumab is associated with lesser cardiac toxicities compared with 1-year trastuzumab. However, cardiac toxicity per se should not be a factor for deciding the duration of adjuvant trastuzumab as cardiac toxicity is mostly reversible. The pros and cons of a longer duration of trastuzumab vis-à-vis cardiac toxicity should be discussed with the patient. The patient can then make an informed decision whether they prefer 1-year trastuzumab due to better DFS and OS or shorter duration of trastuzumab due to the increased risk of cardiac toxicity with 1-year adjuvant trastuzumab. The treatment of early breast cancer has evolved from the time of recruitment of these trials. Today's emphasis is on nonanthracycline-based adjuvant regimens, especially in low-risk, node-negative, estrogen receptor (ER)-positive patients which probably negates the additional cardiotoxicity seen with 12 months of trastuzumab when combined with anthracycline-based regimens of the trial eras. ${ }^{17}$ We need more trials of adjuvant trastuzumab with nonanthracycline-based regimens to see the effectiveness of a shorter duration of trastuzumab. ${ }^{18}$

Individually five out of six trials failed to prove noninferiority of shorter duration of trastuzumab against 1-year trastuzumab. However, overall, the meta-analysis was able to show noninferiority for DFS and OS albeit with a $25 \%$ possibility of inferiority at upper $\mathrm{CI} .{ }^{11}$ The authors have discussed the strengths and limitations of their meta-analysis in detail in their paper. ${ }^{11}$ I would echo their concerns in that the trials included in their meta-analysis are heterogeneous with regard to the time of randomization, chemotherapy drugs, and scheduled used, and follow-up period. Therefore, even 
if we agree that a shorter duration of trastuzumab is enough we do not know the ideal chemotherapy backbone for it. However, the majority of the neoadjuvant and adjuvant trastuzumab trials have used docetaxel at a dose of $100 \mathrm{mg} / \mathrm{m}^{2} \mathrm{q}$ 3 weekly as a combination chemotherapy backbone. ${ }^{17}$

The authors could not show a benefit of shorter duration of trastuzumab in a subgroup analysis of patient age ( $<$ or $>50$ years), ER status, axillary nodal status, stage, and timing of trastuzumab (sequential or concurrent with chemotherapy). ${ }^{11}$ However, the authors concluded that 1 -year trastuzumab is superior to shorter duration trastuzumab in patients who are older than 50 years, are ER-negative, and receive concomitant trastuzumab as the lower limit of $95 \%$ $\mathrm{CI}$ did not cross unity. These subgroups analyses were not preplanned in the individual trials and therefore their results should be taken as hypothesis generating and not practice changing. We also need a longer follow-up in the ER-positive subgroup to assess the benefit of adjuvant trastuzumab as relapses in this subgroup can occur late. ${ }^{18}$ Surprisingly heavy nodal positivity did not influence the outcome. One reason for this could be that the authors defined pathologically staged N3 axillary lymph node as $\geq 4$ nodes instead of the American Joint Committee on Cancer (AJCC) staging that defines $\mathrm{N} 3$ as $\geq 10$ nodes.

Adjuvant anti-HER2 therapy itself has evolved over the trial periods with additions of newer molecules like pertuzumab (APHINITY trial) and the concept of dual HER2 blockade in the adjuvant setting and extended HER2 blockade with neratinib after 1 year of adjuvant trastuzumab (ExteNET trial). ${ }^{19,20}$ Therefore, the question of 6 versus 12 months of a single anti HER2 agent is probably losing its relevance.

A similar meta-analysis was published by Chen et al in 2019 with the same patient cohort. ${ }^{21}$ However, they demonstrated that 1-year adjuvant trastuzumab was superior to a shorter duration of adjuvant trastuzumab. Both DFS (HR: 1.13 ; 95\% CI: $1.03-1.25 ; p=0.01$ ) and OS (HR: 1.16; 95\% CI: $1.01-1.32 ; p=0.03)$ were significantly improved with conventional 1-year trastuzumab treatment compared with shorter treatments. This is opposite to the observation of Gulia et al. ${ }^{11}$ All it indicates that meta-analysis of pooled data done retrospectively may vary as per the way the observer wants to see it. Chen et al did not do an IPD meta-analysis, unlike Gulia et al. ${ }^{11,21}$

The million-rupee question is do we change our practice when we go to our clinics on Monday morning based on the results of the meta-analysis of Gulia et al. ${ }^{11}$ The answer is both yes and no. Yes, 6-month adjuvant trastuzumab is noninferior to 1-year adjuvant trastuzumab for DFS. Yes, that the financial toxicity of a shorter regimen is lesser, and in a resource-limited setting the incremental benefits seen with a longer duration of trastuzumab does not justify the cost. No, we do not know which subgroup of patients will benefit from shorter or 1-year trastuzumab. No, a 9-12-week trastuzumab schedule cannot replace a 6-month schedule. However, due to financial constraints if only giving 9- to 12-week trastuzumab is possible then this is better than giving no trastuzumab as has been reported from studies in India. ${ }^{22-24}$ The patients should be explained the pros and cons of 1-year and shorter trastuzumab and their views also need to be considered when recommending the treatment.

Based on subgroup analysis, the short course trastuzumab of 6 months has some role in low-risk early breast cancer patients with ER-positive, node-negative, and small tumor size and will be beneficial from a cost-effectiveness point, especially in low- and middle-income countries (LMICs) like India. Such a strategy may encourage more and more patients from LMICs to take trastuzumab and get benefited from it in an adjuvant setting.

Patients with tumor size less than $3 \mathrm{~cm}$ and with node-negative HER2-positive disease planned for chemotherapy with single-agent weekly paclitaxel for 12 cycles should receive adjuvant trastuzumab for 1-year as per the Adjuvant Paclitaxel Trastuzumab (APT) trial design. ${ }^{25}$

Unless we are clear what is truly acceptable as a noninferiority margin, we might never come to a consensus whether shorter duration trastuzumab is enough, till then we should continue to use our clinical judgment based on the resources we have and the patient's disease burden.

\section{Funding \\ None.}

\section{Conflict of Interest}

None declared.

\section{References}

1 Piccart-Gebhart MJ, Procter M, Leyland-Jones B, et al. Herceptin Adjuvant (HERA) Trial Study Team. Trastuzumab after adjuvant chemotherapy in HER2-positive breast cancer. N Engl J Med 2005;353(16):1659-1672

2 Romond EH, Perez EA, Bryant J, et al. Trastuzumab plus adjuvant chemotherapy for operable HER2-positive breast cancer. N Engl J Med 2005;353(16):1673-1684

3 Slamon D, Eiermann $\mathrm{W}$, Robert $\mathrm{N}$, et al. Breast Cancer International Research Group. Adjuvant trastuzumab in HER2-positive breast cancer. $\mathrm{N}$ Engl J Med 2011;365(14):1273-1283

4 Moja L, Tagliabue L, Balduzzi S, et al. Trastuzumab containing regimens for early breast cancer. Cochrane Database Syst Rev 2012;2012(4):CD006243

5 Joensuu H, Kellokumpu-Lehtinen PL, Bono P, et al. FinHer Study Investigators. Adjuvant docetaxel or vinorelbine with or without trastuzumab for breast cancer. $\mathrm{N}$ Engl J Med 2006;354(8):809-820

6 Conte P, Frassoldati A, Bisagni G, et al. Nine weeks versus 1 year adjuvant trastuzumab in combination with chemotherapy: final results of the phase III randomized Short-HER study. Ann Oncol 2018;29(12):2328-2333

7 Joensuu $\mathrm{H}$, Fraser J, Wildiers $\mathrm{H}$, et al. Effect of adjuvant trastuzumab for a duration of 9 weeks vs 1 year with concomitant chemotherapy for early human epidermal growth factor receptor 2-positive breast cancer: the SOLD randomized clinical trial. JAMA Oncol 2018;4(9):1199-1206

8 Mavroudis D, Saloustros E, Malamos N, et al. Breast Cancer Investigators of Hellenic Oncology Research Group (HORG), Athens, Greece. Six versus 12 months of adjuvant trastuzumab in combination with dose-dense chemotherapy for women with HER2-positive breast cancer: a multicenter randomized study by the Hellenic Oncology Research Group (HORG. Ann Oncol 2015;26(7):1333-1340 
9 Pivot X, Romieu G, Debled M, et al. PHARE trial investigators. 6 months versus 12 months of adjuvant trastuzumab in early breast cancer (PHARE): final analysis of a multicentre, open-label, phase 3 randomised trial. Lancet 2019;393(10191) :2591-2598

10 Earl HM, Hiller L, Vallier AL, et al. PERSEPHONE Steering Committee and Trial Investigators. 6 versus 12 months of adjuvant trastuzumab for HER2-positive early breast cancer (PERSEPHONE): 4-year disease-free survival results of a randomised phase 3 non-inferiority trial. Lancet 2019;393(10191): 2599-2612

11 Gulia S, Kannan S, Badwe R, Gupta S. Evaluation of 1-year vs shorter durations of adjuvant trastuzumab among patients with early breast cancer: an individual participant data and trial-level meta-analysis. JAMA Netw Open 2020;3(8):e2011777

12 Drevon D, Fursa SR, Malcolm AL. Intercoder reliability and validity of WebPlotDigitizer in extracting graphed data. Behav Modif 2017;41(2):323-339

13 Althunian TA, de Boer A, Groenwold RHH, Klungel OH. Defining the noninferiority margin and analysing noninferiority: an overview. Br J Clin Pharmacol 2017;83(8):1636-1642

14 Marseille E, Larson B, Kazi DS, Kahn JG, Rosen S. Thresholds for the cost-effectiveness of interventions: alternative approaches. Bull World Health Organ. 2015;93(2):118-24.

15 Gupta N, Verma RK, Gupta S, Prinja S. Cost effectiveness of trastuzumab for management of breast cancer in India. JCO Glob Oncol 2020;6:205-21610.1200/JG0.19.00293

16 Goldhirsch A, Gelber RD, Piccart-Gebhart MJ, et al. Herceptin Adjuvant (HERA) Trial Study Team. 2 years versus 1 year of adjuvant trastuzumab for HER2-positive breast cancer (HERA): an open-label, randomised controlled trial. Lancet 2013;382(9897):1021-1028
17 Jasra S, Anampa J. Anthracycline use for early stage breast cancer in the modern era: a review. Curr Treat Options Oncol 2018;19(6):30

18 Hurvitz SA. Is the duration of adjuvant trastuzumab debate still clinically relevant? Lancet 2019;393(10191):2565-2567

19 von Minckwitz G, Procter M, de Azambuja E, et al; APHINITY Steering Committee and Investigators. Adjuvant pertuzumab and trastuzumab in early HER2-positive breast cancer. $\mathrm{N}$ Engl J Med 2017;377(2):122-131

20 Martin M, Holmes FA, Ejlertsen B, et al. ExteNET Study Group. Neratinib after trastuzumab-based adjuvant therapy in HER2-positive breast cancer (ExteNET): 5-year analysis of a randomised, double-blind, placebo-controlled, phase 3 trial. Lancet Oncol 2017;18(12):1688-1700

21 Chen L, Zhou W, Hu X, Yi M, Ye C, Yao G. Short-duration versus 1-year adjuvant trastuzumab in early HER2 positive breast cancer: a meta-analysis of randomized controlled trials. Cancer Treat Rev 2019;75:12-19

22 Sethjiwala T, Dhanushkodi M, Radhakrishnan V, et al. Adjuvant short-course trastuzumab in breast cancer. Indian J Gynecol Oncolog 2019;17:68

23 Hingmire SS, Sambhus MB, Kelkar DS, Joshi S, Parikh PM, Bharath R. Efficacy and safety of short course adjuvant trastuzumab combination chemotherapy in breast cancer. South Asian J Cancer 2017;6(2):47-50

24 Manuprasad A, Shenoy PK, Jones J, Vinin NV, Dharmarajan A, Muttath G. Short-course adjuvant trastuzumab in breast cancer: experience from a tertiary cancer center in rural India. Cancer Res Stat Treat 2020;3:69-73

25 Tolaney SM, Barry WT, Dang CT, et al. Adjuvant paclitaxel and trastuzumab for node-negative, HER2-positive breast cancer. N Engl J Med 2015;372(2):134-141 